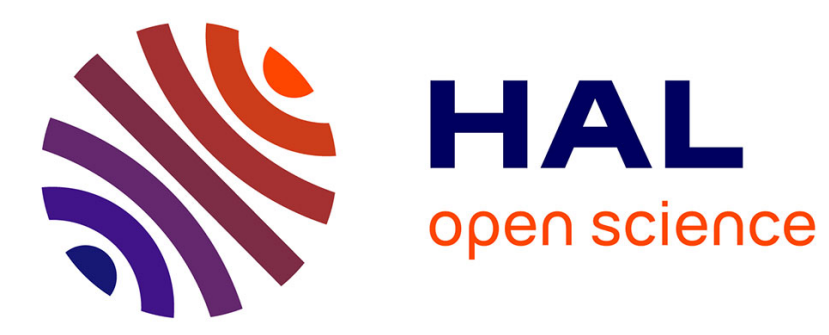

\title{
Nucleation of twisted and tubular states in chiral ribbons
}

Ralf Blossey

\section{To cite this version:}

Ralf Blossey. Nucleation of twisted and tubular states in chiral ribbons. Physical Review E , 2017, Physical Review. E, 96 (3-1), pp.032405. 10.1103/PhysRevE.96.032405 . hal-03203795

\section{HAL Id: hal-03203795 \\ https://hal.univ-lille.fr/hal-03203795}

Submitted on 19 May 2021

HAL is a multi-disciplinary open access archive for the deposit and dissemination of scientific research documents, whether they are published or not. The documents may come from teaching and research institutions in France or abroad, or from public or private research centers.
L'archive ouverte pluridisciplinaire HAL, est destinée au dépôt et à la diffusion de documents scientifiques de niveau recherche, publiés ou non, émanant des établissements d'enseignement et de recherche français ou étrangers, des laboratoires publics ou privés. 


\title{
Nucleation of twisted and tubular states in chiral ribbons
}

\author{
Ralf Blossey \\ University of Lille 1, Unité de Glycobiologie Structurale et Fonctionnelle, CNRS UMR8576, 59000 Lille, France
}

(Received 2 March 2017; published 7 September 2017)

\begin{abstract}
Bilayers of chiral molecules can self-assemble into twisted and tubular structures, as was recently shown with chiral molecular constituents such as ssDNA-amphiphiles. I show that the dynamics of the transition between these topologies is driven by a nucleation mechanism that bears a striking formal similarity to that encountered in first-order wetting and dewetting transitions. Exploiting this analogy enables the critical nuclei of the transition to be calculated.
\end{abstract}

DOI: 10.1103/PhysRevE.96.032405

\section{INTRODUCTION}

DNA remains the most interesting material for selfassembly, with numerous variations that have appeared in the literature over the past years. Research has mostly been concerned with the construction of complex topologies, and less with the dynamical processes underlying the selfassembly - whose knowledge, however, is crucial in order to obtain correct structures or sufficient yields. This has been highlighted recently by the work by Jacobs et al., who demonstrated the importance of nucleation kinetics in DNA-brick construction [1].

A different class of DNA-based self-assembly processes falls into the class of the statistical mechanics of ribbons, itself conceptually fitting into the emerging paradigm of geometrically frustrated (self-)assembly [2]. Much work has been devoted to the morphological states of ribbons [3-7], while again the explicit dynamics of the transitions between the morphologies is far less developed. Of particular interest in this field are chiral ribbons, for which the general equilibrium theory was already developed [8-10]. Several years ago, Ghafouri and Bruinsma [11] showed that a ribbon made of a molecular bilayer of a chiral lipid can display twisted or tubular ribbon structures depending on its width $w$. This result was derived from a wormlike chain-type model for the bilayer. Subsequent experiments by Ziserman et al. [12] were able to validate this result experimentally. Recently, an ssDNA-amphiphile system was shown also to exhibit these ribbon states [13]; this motivated the present work. Another relevant experimental case may be $\beta$-amyloid fibrils $[14,15]$.

Figure 1 sums up the structural details of the system studied in Ref. [13] as an exemplary case. The ssDNA-amphiphile constructs shown in Fig. 1(a) build up the ribbon (bilayer) in the indicated manner, and they produce structures of twisted and tubular ribbons.

The transition between these generic morphologies was studied recently using both microscopic and continuum approaches $[16,17]$. In this work, I highlight an interesting and unexpected analogy of the ribbon-state transition to first-order wetting and dewetting transitions. The knowledge gained in this field can therefore be exploited to deduce key features of ribbon transitions. In the following section, I first develop a phenomenological continuum theory of the equilibrium states of the ribbons, building on the results from [11] and [16].

\section{EQUILIBRIUM RIBBON STATES}

As the starting point of the construction of the equilibrium states, I take the ribbon free energy per unit ribbon length $L$, which I denote by $F(w) . F(w)$ should have a shape similar to the elastic energy $E(w)$ calculated by Ghafouri and Bruinsma (see their Fig. 2) [11]. $E(w)$ has two minima, one at a finite value of $w$, which is identified with the ribbon state, and one at infinite $w$, identified with the tubule state, as seen in Fig. 2. The relative height of the two minima can change by changing the Föppl-van Kármán parameter, which we effectively parametrize as a function of temperature. Note that in [11], the ribbon width is not allowed to change by the addition of particles. For this we need to add a chemical potential term, hence we consider

$$
F(w)=E(w)-\Delta \mu w .
$$

The definition of the chemical potential difference (per unit area) can be found in [16]. As the insert of Fig. 3 in their paper shows, the derivative of the free energy $d F(w) / d w$ should be a constant at both small and large values of $w$, while $E(w) \rightarrow 0$ from [11]. Thus, the definition of $\Delta \mu$ must contain the limiting behavior of the elastic energy of the wide ribbon as defined in [16],

$$
E_{\text {wide }}=\left(\frac{Y k_{0}^{2} t^{3}}{24}-\frac{\mu_{0}}{a^{2}}\right) w,
$$

where $Y$ is Young's modulus, $k_{0}$ the curvature of the ribbon, and $t$ is the square root of the ratio of bending to stretching modulus. The comparison of this expression with the one in [11] thus shows that, in order to match both expressions, $\Delta \mu$ in Eq. (1) must be chosen as

$$
\Delta \mu \equiv \frac{\mu_{0}}{a^{2}}-\frac{Y k_{0}^{2} t^{3}}{24},
$$

which also has the correct dimensions, with $\mu_{0}$ having dimensions of energy.

The equilibrium states of twisted and tubular ribbons as a function of chemical potential described by Armon et al. can be recovered from the condition $d F(w) / d w=0$ yielding the equation

$$
\frac{d E(w)}{d w}=\Delta \mu .
$$

The ribbon states can be located as follows in the phase diagram of Fig. 3. For $\Delta \mu>0$, the free energy is unbounded 
(a)<smiles>[IH2]</smiles>

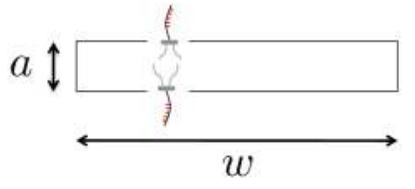

(b)
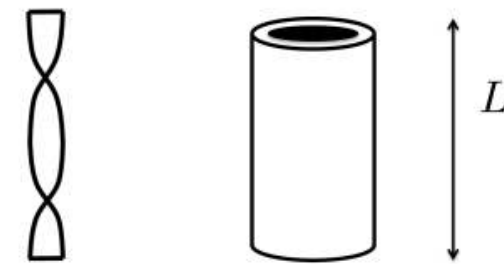

FIG. 1. (a) Schematics of an ssDNA-decorated amphiphile and the placement of the amphiphiles in the bilayer in a cross-section view; (b) the resulting ribbon topologies, namely the twisted and the tubular ribbon.

for large $w$ such that only tubular states exist for any temperature. Physically, this means that at any oversaturation in particles, the ribbons will always close to tubules. Along the transition line $\Delta \mu=0$, for $T<T_{t}$, the minimum at $w=w_{0}$ will lie lower than the minimum at $w=\infty: E(w)<E(\infty)$, such that the twisted ribbon state is stable. For $T>T_{t}$, the reverse situation applies. For the case of undersaturation, $\Delta \mu<0$, the chemical potential term rises linearly for large $w$ such that the free energy per unit ribbon length $F(w)$ in general has two minima. As in [16], these states exist in a range of chemical potential values, bounded by the dashed lines in Fig. 3, which in fact correspond to a spinodal region in which $F(w)$ has two minima of different depth; they have the same depth only at an off-coexistence line. Note that the unfolding of this full phase diagram is not given in [16], as their parametrization of narrow and wide ribbons does not allow to capture the change in the relative depths of the two minima of $F(w)$ due to the second parameter, here identified as temperature; when the coexistence line is crossed at a fixed

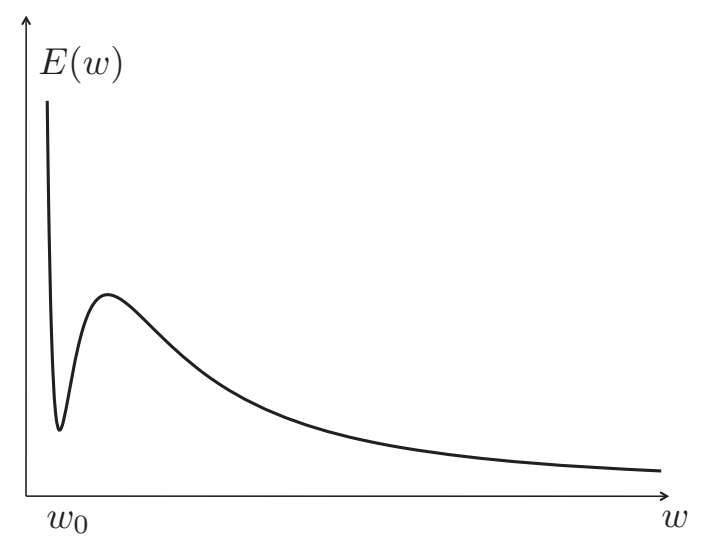

FIG. 2. Schematic ribbon free energy $E(w)$ as obtained from the WLC model in [11]. The free energy describes the case when the tubular ribbon is stable since $E(w=\infty)<E\left(w_{0}\right)$ with $w_{0}$ as the location of the minimum at small $w$.

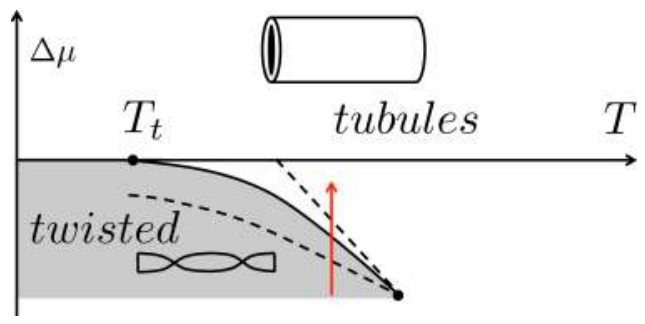

FIG. 3. Phase diagram of the ribbon morphologies, based on the minima of $E(w)-\Delta \mu w$. For $\Delta \mu \geqslant 0, T>T_{t}$, the tubular ribbons are the stable configurations. For $\Delta \mu \leqslant 0, T<T_{t}$, narrow twisted ribbons are stable; at the transition line for $\Delta \mu<0, T>T_{t}$, twisted and tubular ribbons exchange stability if the chemical potential or temperature is changed to cross the line (from the shaded into the white area or reverse); a path at constant $T$ is indicated by an arrow. The transition line is bounded by a spinodal region, indicated by dashed lines, within which $F(w)$ has two minima, corresponding to a stable twisted ribbon and a metastable tubular ribbon state in the shaded area, and a metastable twisted ribbon and a stable tubular ribbon in the white area. Outside of the dashed lines, $F(w)$ only has one minimum, i.e., either a twisted ribbon or a tubular ribbon in the corresponding regions of the phase diagram.

temperature $T>T_{t}$, the transition is from a twisted to a tubular ribbon, passing through the spinodal region.

The shape of $E(w)$ in Fig. 2 bears a striking similarity to the free energy per unit area of a flat film of thickness $f$ above a solid substrate, commonly denoted as the effective interface potential $V(f)$, which dimensionally is a free energy per unit area. (For authoritative and exhaustive introductions into wetting physics, see the reviews in [18] and [19].) Both $E(w)$ and $V(f)$ have a double-well shape with one minimum at a finite value, separated by a barrier from a minimum at infinity. For the wetting case, the minimum at finite $f$ corresponds to the nonwet substrate covered at most by a microscopic film, while the infinitely thick film corresponds to a macroscopic (complete) wetting layer. In analogy with the wetting case, the deviation from complete wetting arises if the film is coupled to a reservoir governed by a corresponding chemical potential, $\Delta \mu$, controlling its thickness. The full potential $\phi(f)=V(f)-\Delta \mu f$ then gives rise to a more symmetric double-well potential, and this holds true in the ribbon case, by analogy. In wetting, the off-coexistence term results in a line of first-order transitions at undersaturation, called the prewetting line, which ends in a critical point of the Ising universality class. As we discuss here ribbon states of finite size, the transitions that arise in the present case are pseudo-phase-transitions, corresponding in fact to transitions in morphologies.

\section{NUCLEATION OF RIBBON STATES}

Having established the basic model free energy and its equilibrium states, we can now turn to the nonequilibrium aspect, i.e., the nucleation of one ribbon state from another. Again, relevant information is available from the wetting case. In wetting films, the complete wetting situation is highly asymmetric with respect to nucleation: going from the nonwet to the wet (or the thin to the thick) film proceeds by the 
nucleation of droplets on the wall, which can be calculated within homogeneous nucleation theory if a proper free-energy functional is assumed, e.g., the effective interface Hamiltonian, $H[f]=\int d^{2} x\left[\frac{\gamma}{2}(\nabla f)^{2}+V(f)-\Delta \mu f\right]$, wherein $\gamma$ is the surface tension of the liquid-vapor interface (the free film surface). The critical nucleus then follows from the variational equation $\delta H / \delta f(r)=0$, with $r$ being the radial coordinate in two dimensions, accompanied by suitable boundary conditions. The reverse direction is essentially blocked for nucleation as the position of the thick film minimum lies at infinity: the corresponding critical hole to be nucleated in a complete wetting film would have an infinite excess free energy. This easily explains why the undercooling of wetting films has always shown long-lived metastable states [20], a fact that has been observed experimentally [21]. Likewise, an undercooling of the tubular state should lead to long-lived metastable tubules.

As for the case of first-order wetting, the nucleation of twisted and tubular ribbons is controlled by the nucleation rate $\Gamma \sim \exp -\left(H\left[w^{*}\right] / k_{B} T\right)$, where $H\left[w^{*}\right]$ is the excess free energy of the critical nucleus of either the twisted or the helicoid phase, with $w^{*}$ denoting the width of the critical nucleus. $H\left[w^{*}\right]$ can be computed by adopting a free-energy expression for an inhomogeneous ribbon. The free energy one can write down for the system is given by

$$
H[w]=\int_{0}^{L} d x\left[\frac{\tau}{2}(\Delta w)^{2}+\frac{\gamma}{2}(\nabla w)^{2}+E[w]-\Delta \mu w\right],
$$

where a line stiffness $\tau$ associated with the ribbon border is introduced, as well as a line or border tension $\gamma$. Further, in
Eq. (5), $L$ is the length of the ribbon, which is supposed to remain unchanged in the transition; dimensionally, we always have $a \ll w \ll L$, where $a$ is the thickness of the ribbon; for an illustration of these lengths, see Fig. 1. To characterize the nuclei of the ribbon transitions, we will be interested in the excess free energy $\Delta H=H\left[w^{*}\right]-H\left[w_{0}\right]$ with $H\left[w_{0}\right]=$ $L F\left(w_{0}\right)$, where, as a reminder, $F$ is a free energy per unit ribbon length.

The profile of the critical nucleus can be determined from the variation of Eq. (5), $\delta H[w] / \delta w=0$, in a one-dimensional geometry, which gives the ribbon nucleus equation

$$
\tau\left(\frac{d^{4} w}{d x^{4}}\right)-\gamma \frac{d^{2} w}{d x^{2}}+\partial_{w} E[w(x)]-\Delta \mu=0 .
$$

The solutions of this equation can be understood by invoking a simpler membrane problem in which the nonlinear terms are first neglected. The membrane equation is then of the wellstudied Helfrich type and reads

$$
\tau\left(\frac{d^{4} w}{d x^{4}}\right)-\gamma \frac{d^{2} w}{d x^{2}}=0 .
$$

Consider the solution of this equation for a membrane spread over a step edge [22]. It is given by the expression

$$
w(x)=A+B \bar{x}+C \cosh (\bar{x})+D \sinh (\bar{x}),
$$

where the constants are fixed by the conditions $w(0)=w^{*}$, $w^{\prime}(0)=0, w\left(L^{*}\right)=0$, and $w^{\prime}\left(L^{*}\right)=0$, where $\bar{x} \equiv x / \lambda$ with $\lambda^{2} \equiv \tau / \gamma$; furthermore, we introduce $\ell \equiv L^{*} / \lambda$. The exact solution is given by

$$
w(\bar{x}) / w^{*}=\left[1-\frac{[\cosh (\ell)-1][\cosh (\bar{x}-1]-\sinh (\ell)[\sinh (\bar{x})-\bar{x}]}{[\cosh (\ell)-1][\cosh (\ell-1]-\sinh (\ell)[\sinh (\ell)-\ell]}\right]
$$

which for $\gamma \rightarrow 0$ becomes

$$
w(x) / w^{*}=1-3\left(\frac{x}{L^{*}}\right)^{2}+2\left(\frac{x}{L^{*}}\right)^{3} .
$$

As the membrane equation (7) is linear, both $w^{*}$ and $L^{*}$ are free parameters. This ceases to be true for the nucleus due to the nonlinearity in $H$.

At this stage, it is worth remembering how the critical nucleus of the wetting problem is computed, which when going from a nonwet to a wet state corresponds to a critical droplet. In $d>2$, the solution to $\delta H[f] / \delta f(r)=0$ has a radial symmetry and is obtained by requiring the initial condition $f(0)=F$ to be found such that $f(\infty)=f_{0}$, the value of a metastable nonwet state. In addition, one requires as a second boundary condition $f^{\prime}(0)=0$. In $d=2$, by virtue of a conserved first integral, the initial condition $f(0)$ is determined by $V(f(0))=V\left(f_{0}\right)$, and the solution has mirror symmetry with respect to its center. In the ribbon case, the nucleus forms at a finite-size object, and the preferred site for its formation will be one of the four corners of the ribbon. The boundary conditions are analogous to the step-edge problem, however now the initial height $w^{*}$ and the value
$L^{*}<L$ must be found such that $w\left(L^{*}\right)=w_{0}$; furthermore, $w^{\prime}(0)=0, w^{\prime}\left(L^{*}\right)=0$. This solution can be found using a shooting argument. Fixing first $w^{*}$, there are two solutions to the equation, one undershooting and one overshooting the minimum of the potential. For each $w^{*}$, there is a value of $L^{*}$ that corresponds to the nucleus solution. The critical nucleus is then obtained from the maximum value of $w^{*}$ for which a finite $L^{*}$ can still be obtained, or, in other words, the maximum in the free energy $H\left[w^{*}\left(L^{*}\right)\right]$. The parameters $w^{*}$ and $L^{*}$ thus play a role analogous to the radius $R$ in the simple droplet model of nucleation in a bulk vapor, or the height $F$ and droplet radius $R$ in wetting droplet and dewetting hole nucleation.

Figure 4 sketches the overall configuration of the nucleus formed at a ribbon. Figure 4(a) corresponds to the nucleation of a helicoidal or tubular state from the twisted nanotape, and Fig. 4(b) corresponds to the reverse case, which can only be realized for $\Delta \mu<0$, i.e., in the situation in which the ribbon has a finite width and has not closed up to a tubule. Qualitatively, the nuclei in both cases are approximately the mirror image of each other. Note that in this drawing the inplane bending of the ribbon in space is ignored, as well as in the model, which is justified in the limit $a \ll w \ll L$, as 

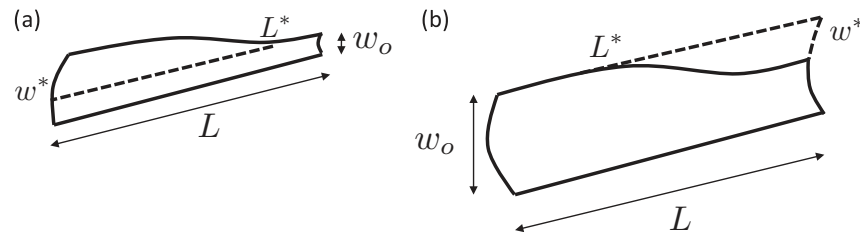

FIG. 4. Schematic drawing of nucleation at a ribbon, going from a small-width ribbon to a large-width ribbon in (a), representing the nucleation of the tubular state from the twisted ribbon, and the inverse case in (b). The initial state of width $w_{0}$ is drawn with broken lines. The bending of the ribbon in space, although ignored in the model, is tentatively indicated.

stated before. Finally, Fig. 5 shows a comparison of the exact result for the Helfrich membrane, Eq. (7), with a numerically computed profile of Eq. (6).

The ribbon nuclei can also be characterized by their scaling behavior in the vicinity of the transition. To obtain this, one has to balance the bending and line tension energy terms against the decay of $E(w)$ at large $w$, which we take as

$$
E(w) \sim w^{1-\sigma}
$$

with $\sigma>1$. For short ribbons, line tension dominates over bending, and one obtains from Eq. (5) the scaling

$$
w^{*} \sim\left(L^{*}\right)^{\frac{2}{\sigma+1}}
$$

while for long ribbons the bending term dominates and one has

$$
w^{*} \sim\left(L^{*}\right)^{\frac{4}{\sigma+1}}
$$

In both case, the excess free energy $\Delta H=H\left[w^{*}\right]-H\left[w_{0}\right]$ scales as $\Delta H \sim L^{*}$, hence with the corresponding different exponent when expressed in terms of $w^{*}$. For the case of a tubular ribbon above the first-order transition point

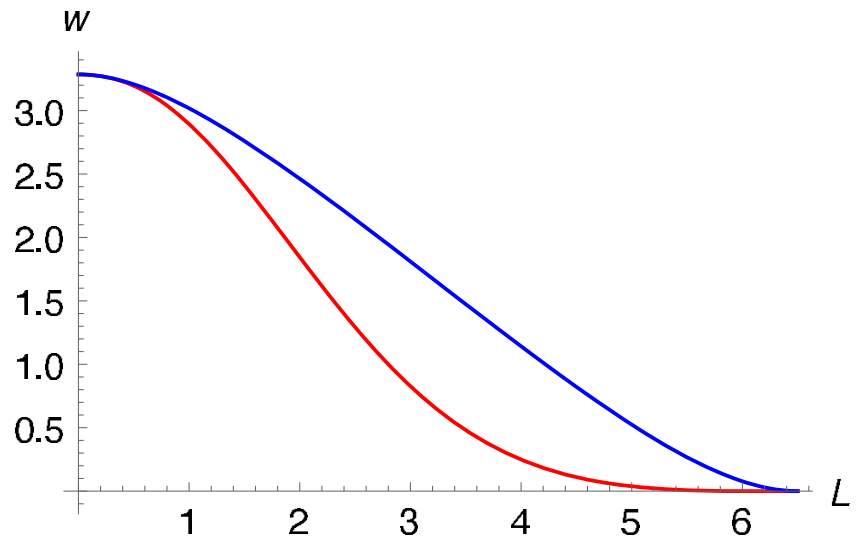

FIG. 5. Ribbon nucleus (red) vs membrane at a step (blue). The energy $E(w)$ is approximated by a cubic polynomial, which is valid away from the transition, when the minimum at $w_{0}$ is not very pronounced (near the spinodal). $E(w)=w^{2}-0.3 w^{3}$ served as the model potential for this case; $\tau=\gamma=1$.

approaching the coexistence line $\Delta \mu=0$ from below, one has in addition the condition $w^{*} \sim \Delta \mu^{-1}$.

To conclude, we have shown that the critical nuclei of the twisted-tubular ribbon transition can be computed from an effective model that bears strong similarities to the effective interface model approach in wetting and dewetting. The dependence of the excess free energy on the ribbon length $L^{*} \sim L$ indicates that this parameter is also relevant for the formation process of the ultimate stable structure (see Ref. [13]). Experiments at different ribbon lengths $L$ would thus be of interest to test the predicted behavior, in particular at undersaturation conditions, $\Delta \mu<0$.

\section{ACKNOWLEDGMENTS}

I thank Fabrizio Cleri for a critical reading of an earlier version of the manuscript. An anonymous referee is thanked for pointing out Ref. [16], and for an enlightening exchange on the nature of the homogeneous minima of $F(w)$.
[1] W. M. Jacobs, A. Reinhardt, and D. Frenkel, Rational design of self-assembly pathways for complex multicomponent structures, Proc. Natl. Acad. Sci. USA 112, 6314 (2015).

[2] G. M. Grason, Perspective: Geometrically-frustrated assemblies, J. Chem. Phys. 145, 110901 (2016).

[3] L. Giomi and L. Mahadevan, Statistical Mechanics of Developable Ribbons, Phys. Rev. Lett. 104, 238104 (2010).

[4] C. Nadir Kaplan, H. Tu, R. A. Pelcovits, and R. B. Mayer, Theory of depletion-induced phase transition from chiral smectic-A twisted ribbon to semi-infinite flat membranes, Phys. Rev. E 82, 021701 (2010).

[5] E. H. Yong and L. Mahadevan, Statistical Mechanics and Shape Transitions in Microscopic Plates, Phys. Rev. Lett. 112, 048101 (2014).

[6] J. Chopin and A. Kudrolli, Helicoids, Wrinkles, and Loops in Twisted Ribbons, Phys. Rev. Lett. 111, 174302 (2013).

[7] J. Chopin, V. Démery, and B. Davidovitch, Roadmap to the morphological instabilities of a stretched twisted ribbon, in The
Mechanics of Ribbons and Möbius Bands, edited by R. Fosdick and E. Fried (Springer, Dordrecht, 2014), pp. 137-189.

[8] D. S. Chung, G. B. Benedek, F. M. Konikoff, and J. M. Donovan, Elastic free energy of anisotropic helical ribbons as metastable intermediates in the crystallization of cholesterol, Proc. Natl. Acad. Sci. USA 90, 11341 (1993).

[9] J. V. Selinger, F. MacKintosh, and J. M. Schnur, Theory of cylindrical tubules and helical ribbons of chiral lipid membranes, Phys. Rev. E 53, 3804 (1996).

[10] S. Komura and O. Y. Zhong-can, High and Low-Pitch Helical Structures of Titled Chiral Lipid Bilayers, Phys. Rev. Lett. 81, 473 (1998).

[11] R. Ghafouri and R. Bruinsma, Helicoid to Spiral Ribbon Transition, Phys. Rev. Lett. 94, 138101 (2005); the authors actually employ a slightly different nomenclature for the ribbon states.

[12] L. Ziserman, A. Mor, D. Harreis, and D. Danino, Curvature Instability in a Chiral Amphiphile Self-Assembly, Phys. Rev. Lett. 106, 238105 (2011). 
[13] T. R. Pearce and E. Kokkoli, DNA nanotubes and helical nanotapes via self-assembly of ssDNA-amphiphiles, Soft Matter 11, 109 (2015).

[14] V. Castelletto, I. W. Hamley, R. A. Hule, and D. Pochan, Helical-ribbon formation by a beta-amino acid modified amyloid beta-peptide fragment, Angew. Chem. Int. Ed. 48, 2317 (2009).

[15] T. P. J. Knowles, A. De Simone, A. W. Fitzpatrick, A. Baldwin, S. Meehan, L. Rajah, M. Vendruscolo, M. E. Welland, C. M. Dobson, and E. M. Terentjev, Twisting Transition Between Crystalline and Fibrillar Phases of Aggregated Peptides, Phys. Rev. Lett. 109, 158101 (2012).

[16] S. Armon, H. Aharoni, M. Moshe, and E. Sharon, Shape selection in chiral ribbons: From seed pods to supramolecular assemblies, Soft Matter 10, 2733 (2014).
[17] M. Gruziel and P. Szymczak, From ribbons to tubules: A computational study of the polymorphism in aggregation of helical filaments, Soft Matter 11, 6294 (2015).

[18] P. G. de Gennes, Wetting: Statics and dynamics, Rev. Mod. Phys. 57, 827 (1985).

[19] D. Bonn, J. Eggers, J. Indekeu, J. Meunier, and E. Rolley, Wetting and spreading, Rev. Mod. Phys. 81, 739 (2009).

[20] R. Bausch and R. Blossey, Lifetime of undercooled wetting layers, Phys. Rev. E 50, R1759(R) (1994).

[21] M. Schick and P. Taborek, Anomalous nucleation at first-order wetting transitions, Phys. Rev. B 46, 7312 (1992).

[22] M. Deserno, Fluid lipid membranes-A primer, available at the author's institutional website, https://www.cmu.edu/biolphys/ deserno/pdf/membrane_theory.pdf. 